International Journal of Pure and Applied Mathematics

Volume 108 No. 4 2016, 819-830

ISSN: 1311-8080 (printed version); ISSN: 1314-3395 (on-line version)

url: http://www.ijpam.eu

doi: $10.12732 /$ ijpam.v108i4.8

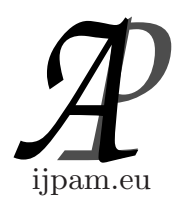

\title{
SOFT $L$-FUZZY QUASI-UNIFORMITIES AND SOFT L-FUZZY TOPOGENOUS ORDERS
}

\author{
Jung Mi Ko${ }^{1}$, Ju-Mok $\mathrm{Oh}^{2}$, Yong Chan $\mathrm{Kim}^{3} \S$ \\ 1,2,3 Department of Mathematics \\ Gangneung-Wonju University \\ Gangneung, Gangwondo 210-702, KOREA
}

\begin{abstract}
In this paper, we introduce soft $L$-fuzzy topogenous orders and soft $L$-fuzzy quasi-uniformities in complete residuated lattice. We obtain two soft $L$-fuzzy bitopogenous structures induced by a soft $L$-fuzzy quasi-uniformity. We give their examples.
\end{abstract}

AMS Subject Classification: 03E72, 06A15, 06F07, 54A05

Key Words: complete residuated lattices, fuzzy soft sets, soft $L$-fuzzy topogenous orders, soft $L$-fuzzy quasi-uniformities

\section{Introduction}

Molodtsov [9] introduced the soft set as a mathematical tool for dealing information as the uncertainty of data in engineering, physics, computer sciences and many other diverse field. Pawlak's rough set $[10,11]$ can be viewed as a special case of soft rough sets [3]. The topological structures of soft sets have been developed by many researchers $[1,2,12-15]$.

On the other hand, Hájek [4] introduced a complete residuated lattice which is an algebraic structure for many valued logic. It is an important mathematical tool for algebraic structure of fuzzy contexts $[4,5]$.

In this paper, we introduce soft $L$-fuzzy topogenous orders and soft $L$ fuzzy quasi-uniformities in complete residuated lattice. From Theorem 14, we obtain two soft $L$-fuzzy bitopogenous structures induced by a soft $L$-fuzzy quasi-

Received: April 3, 2016

Published: August 16, 2016

$\S$ Correspondence author (c) 2016 Academic Publications, Ltd.

url: www.acadpubl.eu 
uniformity. We give their examples.

\section{Preliminaries}

Definition 1. [4,5] An algebra $(L, \wedge, \vee, \odot, \rightarrow, 0,1)$ is called a complete residuated lattice if it satisfies the following conditions:

(C1) $L=(L, \leq, \vee, \wedge, 1,0)$ is a complete lattice with the greatest element 1 and the least element 0 ;

(C2) $(L, \odot, 1)$ is a commutative monoid;

(C3) $x \odot y \leq z$ iff $x \leq y \rightarrow z$ for $x, y, z \in L$.

In this paper, we assume that $\left(L, \leq, \odot, \rightarrow, \oplus,^{*}\right)$ is a complete residuated lattice with an order reversing involution ${ }^{*}$ which is defined by $x \oplus y=$ $\left(x^{*} \odot y^{*}\right)^{*}$ unless otherwise specified and we denote $L_{0}=L-\{0\}$.

Lemma 2. $[4,5]$ For each $x, y, z, x_{i}, y_{i}, w \in L$, we have the following properties.

(1) $1 \rightarrow x=x, 0 \odot x=0, x \rightarrow y=1$ iff $x \leq y$.

(2) If $y \leq z$, then $x \odot y \leq x \odot z, x \oplus y \leq x \oplus z, x \rightarrow y \leq x \rightarrow z$ and $z \rightarrow x \leq y \rightarrow x$.

(3) $x \odot y \leq x \wedge y \leq x \vee y \leq x \oplus y$.

(4) $\left(\bigwedge_{i} y_{i}\right)^{*}=\bigvee_{i} y_{i}^{*},\left(\bigvee_{i} y_{i}\right)^{*}=\bigwedge_{i} y_{i}^{*}$,

(5) $x \odot\left(\bigvee_{i} y_{i}\right)=\bigvee_{i}\left(x \odot y_{i}\right)$

(6) $x \oplus\left(\bigwedge_{i} y_{i}\right)=\bigwedge_{i}\left(x \oplus y_{i}\right)$.

(7) $x \rightarrow\left(\bigwedge_{i} y_{i}\right)=\bigwedge_{i}\left(x \rightarrow y_{i}\right)$.

(8) $\left(\bigvee_{i} x_{i}\right) \rightarrow y=\bigwedge_{i}\left(x_{i} \rightarrow y\right)$.

(9) $x \rightarrow\left(\bigvee_{i} y_{i}\right) \geq \bigvee_{i}\left(x \rightarrow y_{i}\right)$

(10) $\left(\bigwedge_{i} x_{i}\right) \rightarrow y \geq \bigvee_{i}\left(x_{i} \rightarrow y\right)$.

(11) $(x \odot y) \rightarrow z=x \rightarrow(y \rightarrow z)=y \rightarrow(x \rightarrow z)$.

(12) $x \odot(x \rightarrow y) \leq y$ and $x \rightarrow y \leq(y \rightarrow z) \rightarrow(x \rightarrow z)$.

(13) $(x \rightarrow y) \odot(z \rightarrow w) \leq(x \odot z) \rightarrow(y \odot w)$.

(14) $(x \rightarrow y) \odot(z \rightarrow w) \leq(x \oplus z) \rightarrow(y \oplus w)$.

(15) $x \rightarrow y \leq(x \odot z) \rightarrow(y \odot z)$ and $(x \rightarrow y) \odot(y \rightarrow z) \leq x \rightarrow z$.

(16) $x \odot y \odot(z \odot w) \leq(x \odot z) \oplus(y \odot w)$.

Definition 3. [7] Let $X$ be an initial universe of objects and $E$ the set of parameters (attributes) in $X$. A pair $(F, A)$ is called a fuzzy soft set over $X$, where $A \subset E$ and $F: A \rightarrow L^{X}$ is a mapping. We denote $S(X, A)$ as the family of all fuzzy soft sets under the parameter $A$. 
Definition 4. [7] Let $(F, A)$ and $(G, A)$ be two fuzzy soft sets over a common universe $X$.

(1) $(F, A)$ is a fuzzy soft subset of $(G, A)$, denoted by $(F, A) \leq(G, A)$ if $F(\epsilon) \leq G(\epsilon)$, for each $\epsilon \in A$.

(2) $(F, A) \wedge(G, A)=(F \wedge G, A)$ if $(F \wedge G)(\epsilon)=F(\epsilon) \wedge G(\epsilon)$ for each $\epsilon \in A$.

(3) $(F, A) \vee(G, A)=(F \vee G, A)$ if $(F \vee G)(\epsilon)=F(\epsilon) \vee G(\epsilon)$ for each $\epsilon \in A$.

(4) $(F, A) \odot(G, A)=(F \odot G, A)$ if $(F \odot G)(\epsilon)=F(\epsilon) \odot G(\epsilon)$ for each $\epsilon \in A$.

(5) $(F, A)^{*}=\left(F^{*}, A\right)$ if $F^{*}(\epsilon)=(F(\epsilon))^{*}$ for each $\epsilon \in A$.

(6) $(F, A) \oplus(G, A)=(F \oplus G, A)$ if $(F \oplus G)(\epsilon)=\left(F^{*}(\epsilon) \odot G^{*}(\epsilon)\right)^{*}$ for each $\epsilon \in A$.

\section{Soft $L$-Fuzzy Quasi Uniformities and Soft $L$-Fuzzy Topogenous Orders}

Definition 5. A mapping $\xi: S(X, A) \times S(X, A) \rightarrow L$ is called a soft $L$-fuzzy semi-topogenous order on $X$ if it satisfies the following axioms.

$(\mathrm{ST} 1) \xi\left(\left(1_{X}, A\right),\left(1_{X}, A\right)\right)=\xi\left(\left(0_{X}, A\right),\left(0_{X}, A\right)\right)=1$.

(ST2) If $\xi((F, A),(G, A)) \neq 0$, then $(F, A) \leq(G, A)$.

(ST3) If $\left(F_{1}, A\right) \leq(F, A), \quad(G, A) \leq\left(G_{1}, A\right)$, then $\xi((F, A),(G, A)) \leq$ $\xi\left(\left(F_{1}, A\right),\left(G_{1}, A\right)\right)$.

A mapping $\xi$ is called a soft strong $L$-fuzzy semi-topogenous order on $X$ if it satisfies (ST1), (ST3) and the following axiom.

(S) $\xi((F, A),(G, A)) \leq S((F, A),(G, A))$ where

$$
S((F, A),(G, A))=\bigwedge_{a \in A} \bigwedge_{x \in X}(F(a)(x) \rightarrow G(a)(x)) .
$$

Remark 6. If $\xi$ is a soft (resp. strong) $L$-fuzzy semi-topogenous order on $X$. Define a mapping $\xi^{s}: S(X, A) \times S(X, A) \rightarrow L$ as $\xi^{s}((F, A),(G, A))=$ $\xi\left((G, A)^{*},(F, A)^{*}\right)$. Then $\xi^{s}$ is a soft (resp. strong) $L$-fuzzy semi-topogenous order on $X$.

Definition 7. A soft (resp. strong) $L$-fuzzy semi-topogenous order $\xi$ is called:

(1) soft (resp. strong) $L$-fuzzy topogenous if (T)

$$
\begin{aligned}
& \xi\left(\left(F_{1}, A\right) \odot\left(F_{2}, A\right),\left(G_{1}, A\right) \odot\left(G_{2}, A\right)\right) \\
& \geq \xi\left(\left(F_{1}, A\right),\left(G_{1}, A\right)\right) \odot \xi\left(\left(F_{2}, A\right),\left(G_{2}, A\right)\right) .
\end{aligned}
$$


(2) soft (resp. strong) $L$-fuzzy cotopogenous if (CT)

$$
\begin{aligned}
& \xi\left(\left(F_{1}, A\right) \oplus\left(F_{2}, A\right),\left(G_{1}, A\right) \oplus\left(G_{2}, A\right)\right) \\
& \geq \xi\left(\left(F_{1}, A\right),\left(G_{1}, A\right)\right) \odot \xi\left(\left(F_{2}, A\right),\left(G_{2}, A\right)\right),
\end{aligned}
$$

(3) soft (resp. strong) $L$-fuzzy bitopogenous if $\xi$ are soft (resp. strong) $L$-fuzzy topogenous and soft (resp. strong) $L$-fuzzy cotopogenous.

Definition 8. A soft (resp. strong) $L$-fuzzy topogenous (resp. cotopogenous) order $\xi$ on $X$ is said to be a soft (resp. strong) $L$-fuzzy topogenous (resp. cotopogenous) structure if $\xi \circ \xi \geq \xi$, where

$$
\begin{aligned}
& (\xi \circ \xi)((F, A),(H, A)) \\
& =\bigvee_{(G, A) \in S(X, A)} \xi((F, A),(G, A)) \odot \xi((G, A),(H, A))
\end{aligned}
$$

Example 9. Let $H=\left\{h_{i} \mid i=\{1, \ldots, 6\}\right\}$ with $h_{i}=$ house and $E=$ $\{e, b, w, c, i\}$ with $e=$ expensive, $b=$ beautiful, $w=$ wooden, $c=$ creative, $i=$ in the green surroundings.

Define a binary operation $\odot$ on $[0,1]$ by

$$
\begin{gathered}
x \odot y=\max \{0, x+y-1\}, x \rightarrow y=\min \{1-x+y, 1\} \\
x \oplus y=\min \{1, x+y\}, x^{*}=1-x
\end{gathered}
$$

Then $([0,1], \wedge, \rightarrow, 0,1)$ is a complete residuated lattice (ref.[4,5]). Let $A=$ $\{b, c, i\} \subset E$ and $X=\left\{h^{1}, h^{4}, h^{5}, h^{6}\right\}$. Put $(H, A)$ be a fuzzy soft set as follow:

$$
\begin{array}{cccccc}
(H, A) & h^{1} & h^{4} & h^{5} & h^{6} & \\
b & 0.5 & 0.6 & 0.2 & 0.6 & \\
c & 0.1 & 0.5 & 0.5 & 0.6 & \\
i & 0.4 & 0.6 & 0.6 & 0.5 & \\
& & & & & \\
(H, A) \odot(H, A) & h^{1} & h^{4} & h^{5} & h^{6} \\
b & & 0.0 & 0.2 & 0.0 & 0.2 \\
c & & 0.0 & 0.0 & 0.0 & 0.2 \\
i & & 0.0 & 0.2 & 0.2 & 0.0 \\
\left(H^{*}, A\right) & h^{1} & h^{4} & h^{5} & h^{6} & \\
b & 0.5 & 0.4 & 0.8 & 0.4 & \\
c & 0.9 & 0.5 & 0.5 & 0.4 & \\
i & 0.6 & 0.4 & 0.4 & 0.5 &
\end{array}
$$




$$
\begin{array}{ccccc}
\left(H^{*}, A\right) \oplus\left(H^{*}, A\right) & h^{1} & h^{4} & h^{5} & h^{6} \\
b & 1.0 & 0.8 & 1.0 & 0.8 \\
c & 1.0 & 1.0 & 1.0 & 0.8 \\
i & 1.0 & 0.8 & 0.8 & 1.0
\end{array}
$$

(1) Define a soft $L$-fuzzy topogenous order $\xi: S(X, A) \times S(X, A) \rightarrow L$ as follows

$$
\begin{aligned}
& \xi((F, A),(G, A)) \\
& = \begin{cases}1, & \text { if }(F, A)=\left(0_{X}, A\right) \text { or }(G, A)=\left(1_{X}, A\right) \\
0.6, & \text { if }(F, A) \leq(H, A) \leq(G, A), \\
& (F, A) \leq \leq(H, A) \odot(H, A) \\
0.3, & \text { if }\left(0_{X}, A\right) \neq(F, A) \leq(H, A) \odot(H, A) \\
& \leq(G, A),(H, A) \not \leq(G, A), \\
0, & \text { otherwise. }\end{cases}
\end{aligned}
$$

But it is not a soft $L$-fuzzy topogenous structure because

$$
\begin{aligned}
& \bigvee_{(F, A) \in S(X, A)}(\xi((H, A) \odot(H, A),(F, A)) \\
& \odot \xi((F, A),(H, A) \odot(H, A)))=0 \\
& \geq \xi((H, A) \odot(H, A),(H, A) \odot(H, A))=0.3 .
\end{aligned}
$$

(2) By Remark 6, we obtain a soft $L$-fuzzy cotopogenous order $\xi^{s}: S(X, A) \times$ $S(X, A) \rightarrow L$ as follows

$$
\begin{aligned}
& \xi^{s}((F, A),(G, A)) \\
& = \begin{cases}1, & \text { if }(F, A)=\left(0_{X}, A\right) \text { or }(G, A)=\left(1_{X}, A\right) \\
0.6, & \text { if }(F, A) \leq(H, A)^{*} \leq(G, A), \\
& (G, A) \ngtr(H, A)^{*} \oplus(H, A)^{*} \\
0.3, & \text { if }(F, A) \leq(H, A)^{*} \oplus(H, A)^{*} \\
& \leq(G, A) \neq\left(1_{X}, A\right),(F, A) \not \leq(H, A)^{*}, \\
0, & \text { otherwise, }\end{cases}
\end{aligned}
$$

But it is not a soft $L$-fuzzy cotopogenous structure because

$$
\begin{aligned}
& \bigvee_{(F, A) \in S(X, A)}\left(\xi^{s}\left((H, A)^{*} \oplus(H, A)^{*},(F, A)\right)\right. \\
& \left.\odot \xi^{s}\left((F, A),(H, A)^{*} \oplus(H, A)^{*}\right)\right)=0 \\
& \geq \xi^{s}\left((H, A)^{*} \oplus(H, A)^{*},(H, A)^{*} \oplus(H, A)^{*}\right)=0.3 .
\end{aligned}
$$

Lemma 10. Define a binary mapping $S: S(X, A) \times S(X, A) \rightarrow L$ by

$$
S((F, A),(G, A))=\bigwedge_{a \in A} \bigwedge_{x \in X}(F(a)(x) \rightarrow G(a)(x)) .
$$


Then the following statements hold.

(1) $(F, A) \leq(G, A)$ iff $S((F, A),(G, A))=1$.

(2) If $(F, A) \leq(G, A)$, then

$$
\begin{aligned}
& S((G, A),(H, A)) \leq S((F, A),(H, A)), \\
& S((H, A),(F, A)) \leq S((H, A),(G, A)) .
\end{aligned}
$$

(3) $S((F, A),(G, A)) \odot S((G, A),(H, A))$

$\leq S((F, A),(H, A))$.

(4) $S((F, A),(G, A)) \odot S((H, A),(K, A))$

$\leq S((F, A) \odot(H, A),(G, A) \odot(K, A))$.

(5) $S((F, A),(G, A)) \odot S((H, A),(K, A))$

$\leq S((F, A) \oplus(H, A),(G, A) \oplus(K, A))$.

(6) $S$ is a soft strong $L$-fuzzy bitopogenous order.

Proof. (1) By Lemma 2(1), we obtain

$$
\begin{aligned}
& S((F, A),(G, A))=\top \\
& \text { iff } \bigwedge_{a \in A} \bigwedge_{x \in X}(F(a)(x) \rightarrow G(a)(x))=\top \\
& \text { iff }(F, A) \leq(G, A) .
\end{aligned}
$$

(2) It is easily proved from the definition of $S$.

(3) By Lemma 2(15), we have

$$
\begin{aligned}
& (F(a)(x) \rightarrow G(a)(x)) \odot(G(a)(x) \rightarrow H(a)(x)) \\
& \leq(F(a)(x) \rightarrow H(a)(x)) .
\end{aligned}
$$

(4) By Lemma 2(13), we have

$$
\begin{aligned}
& (F(a)(x) \rightarrow G(a)(x)) \odot(H(a)(x) \rightarrow K(a)(x)) \\
& \leq(F(a)(x) \odot H(a)(x) \rightarrow G(a)(x) \odot H(a)(x)) .
\end{aligned}
$$

(5) By Lemma 2(14), we have

$$
\begin{aligned}
& (F(a)(x) \rightarrow G(a)(x)) \odot(H(a)(x) \rightarrow K(a)(x)) \\
& \leq(F(a)(x) \oplus H(a)(x) \rightarrow G(a)(x) \oplus H(a)(x)) .
\end{aligned}
$$

(6) We easily prove it from (1)-(5).

Definition 11. A mapping $\mathcal{U}: S(X \times X, A) \rightarrow L$ is called a soft $L$-fuzzy pre-uniformity on $X$ iff it satisfies the properties.

(SU1) There exists $(U, A) \in S(X \times X, A)$ such that $\mathcal{U}((U, A))=1$, 
(SU2) If $(V, A) \leq(U, A)$, then $\mathcal{U}((V, A)) \leq \mathcal{U}((U, A))$,

(SU3) For every $(U, A),(V, A) \in S(X \times X, A)$,

$$
\mathcal{U}((U, A) \odot(V, A)) \geq \mathcal{U}((U, A)) \odot \mathcal{U}((V, A))
$$

(SU4) If $\mathcal{U}((U, A)) \neq 0$, then $\left(1_{\triangle}, A\right) \leq(U, A)$, where

$$
1_{\Delta}(x, y)= \begin{cases}1 & \text { if } x=y \\ 0 & \text { if } x \neq y\end{cases}
$$

A soft $L$-fuzzy pre-uniformity $\mathcal{U}$ is called a soft $L$-fuzzy quasi-uniformity if

(Q) $\mathcal{U}(U, A) \leq \bigvee\{\mathcal{U}((V, A)) \odot \mathcal{U}((W, A)) \mid(V, A) \circ(W, A) \leq(U, A)\}$,

where, for all $x, y \in X, a \in A$,

$$
(V(a) \circ W(a))(x, y)=\bigvee_{z \in X}(V(a)(z, x) \odot W(a)(x, y)) .
$$

The triple $(X, A, \mathcal{U})$ is called a soft $L$-fuzzy pre-(resp. quasi-) uniform space.

Remark 12. Let $(X, A, \mathcal{U})$ be a soft $L$-fuzzy pre-uniform space, then by (SU1) and (SU2), we have $\mathcal{U}\left(1_{X \times X}\right)=1$ because $(U, A) \leq\left(1_{X \times X}, A\right)$ for all $(U, A) \in S(X \times X, A)$.

Lemma 13. Let $(X, A, \mathcal{U})$ be a soft $L$-fuzzy pre-uniform space. For each $(U, A) \in S(X \times X, A)$ and $(F, A) \in S(X, A)$, we define, for all $x \in X, a \in A$,

$$
\begin{aligned}
(U, A)[(F, A)](a)(x) & =\bigvee_{y \in X}(F(a)(y) \odot U(a)(y, x)), \\
(U, A)[[(F, A)]](a)(x) & =\bigvee_{y \in X}(F(a)(y) \odot U(a)(x, y)) .
\end{aligned}
$$

For each $(U, A),(V, A),\left(U_{1}, A\right),\left(U_{2}, A\right) \in S(X \times X, A)$ and $(F, A),(G, A)$, $\left(F_{1}, A\right),\left(F_{2}, A\right),\left(F_{i}, A\right) \in S(X, A)$, the following properties hold.

(1) $\operatorname{For} \mathcal{U}((U, A))>0,(F, A) \leq(U, A)[(F, A)]$ and $(F, A) \leq(U, A)[[(F, A)]]$.

(2) $(U, A) \leq(U, A) \circ(U, A)$, for $\mathcal{U}((U, A))>0$.

(3) $((V, A) \circ(U, A))[(F, A)]=(V, A)[(U, A)[(F, A)]]$

$((V, A) \circ(U, A))[[(F, A)]]=(V, A)[[(U, A)[[(F, A)]]]]$.

(4) $(U, A)\left[\bigvee_{i}\left(F_{i}, A\right)\right]=\bigvee_{i}(U, A)\left[\left(F_{i}, A\right)\right]$ and

$(U, A)\left[\left[\bigvee_{i}\left(F_{i}, A\right)\right]\right]=\bigvee_{i}(U, A)\left[\left[\left(F_{i}, A\right)\right]\right]$.

(5) $\left(\left(U_{1}, A\right) \odot\left(U_{2}, A\right)\right)\left[\left(F_{1}, A\right) \odot\left(F_{2}, A\right)\right] \leq\left(U_{1}, A\right)\left[\left(F_{1}, A\right)\right] \odot\left(U_{2}, A\right)\left[\left(F_{2}\right.\right.$, $A)]$. 
(6) $\left(\left(U_{1}, A\right) \odot\left(U_{2}, A\right)\right)\left[\left[\left(F_{1}, A\right) \odot\left(F_{2}, A\right)\right]\right] \leq\left(U_{1}, A\right)\left[\left[\left(F_{1}, A\right)\right]\right] \odot\left(U_{2}, A\right)\left[\left[\left(F_{2}\right.\right.\right.$, A)]].

(7) $\left(\left(U_{1}, A\right) \odot\left(U_{2}, A\right)\right)\left[\left(F_{1}, A\right) \oplus\left(F_{2}, A\right)\right] \leq\left(U_{1}, A\right)\left[\left(F_{1}, A\right)\right] \oplus\left(U_{2}, A\right)\left[\left(F_{2}\right.\right.$,

A)].

(8) $\left(\left(U_{1}, A\right) \odot\left(U_{2}, A\right)\right)\left[\left[\left(F_{1}, A\right) \oplus\left(F_{2}, A\right)\right]\right] \leq\left(U_{1}, A\right)\left[\left[\left(F_{1}, A\right)\right]\right] \oplus\left(U_{2}, A\right)\left[\left[\left(F_{2}\right.\right.\right.$, A)]].

Proof. (1) Since $\mathcal{U}((U, A))>0$, by (U4), $\left(1_{\triangle}, A\right) \leq(U, A)$. It implies $(F, A)=\left(1_{\triangle}, A\right)[(F, A)] \leq(U, A)[(F, A)]$.

(2) Since $\left(1_{\triangle}, A\right) \leq(U, A)$ from $(1),(U, A)=\left(1_{\triangle}, A\right) \circ(U, A) \leq(U, A) \circ$ $(U, A)$.

(3) By Lemma 2 (5), we have

$$
\begin{aligned}
& ((V, A) \circ(U, A))[(F, A)](a)(x) \\
& =\bigvee_{y \in X}\{F(a)(y) \odot((V, A) \circ(U, A))(a)(y, x)\} \\
& =\bigvee_{y \in X}\left\{F(a)(y) \odot \bigvee_{z \in X}(U(a)(y, z) \odot V(a)(z, x))\right\} \\
& =\bigvee_{y \in X} \bigvee_{z \in X}\{F(a)(y) \odot U(a)(y, z) \odot V(a)(z, x)\} \\
& =\bigvee_{z \in X}\left\{\bigvee_{y \in X}(F(a)(y) \odot U(a)(y, z)) \odot V(a)(z, x)\right\} \\
& =\bigvee_{z \in X}\{(U, A)[(F, A)](a)(z) \odot V(a)(z, x)\} \\
& =(V, A)[(U, A)[(F, A)]](a)(x) .
\end{aligned}
$$

(4) By Lemma 2 (5), we have

$$
\begin{aligned}
& (U, A)\left[\bigvee_{i}\left(F_{i}, A\right)\right](a)(x)=\bigvee_{y \in X}\left\{\left(\bigvee_{i} F_{i}(a)(y) \odot U(a)(y, x)\right\}\right. \\
& \left.=\bigvee_{y \in X}\left\{\bigvee_{i}\left\{F_{i}(a)(y) \odot U(a)(y, x)\right)\right\}\right\} \\
& =\bigvee_{i}\left\{\bigvee_{y \in X}\left\{F_{i}(a)(y) \odot U(a)(y, x)\right\}\right\}=\bigvee_{i}(U, A)\left[\left(F_{i}, A\right)\right](a)(x)
\end{aligned}
$$

(5)

$$
\begin{aligned}
& \left(U_{1}, A\right)\left[\left(F_{1}, A\right)\right](a)(x) \odot\left(U_{2}, A\right)\left[\left(F_{2}, A\right)\right](a)(x) \\
& =\left(\bigvee_{y \in X}\left(F_{1}(a)(y) \odot U_{1}(a)(y, x)\right)\right) \\
& \odot\left(\bigvee_{z \in X}\left(F_{2}(a)(z) \odot U_{2}(a)(z, x)\right)\right) \\
& \geq \bigvee_{y \in X}\left(\left(F_{1}(a)(y) \odot U_{1}(a)(y, x)\right) \odot\left(F_{2}(a)(y) \odot U_{2}(a)(y, x)\right)\right) \\
& =\bigvee_{y \in X}\left(\left(\left(F_{1}, A\right) \odot\left(F_{2}, A\right)\right)(a)(y) \odot\left(\left(U_{1}, A\right) \odot\left(U_{2}, A\right)\right)(a)(y, x)\right) \\
& =\left(\left(U_{1}, A\right) \odot\left(U_{2}, A\right)\right)\left[\left(F_{1}, A\right) \odot\left(F_{2}, A\right)\right](a)(x) .
\end{aligned}
$$


(7)

$$
\begin{aligned}
& \left(U_{1}, A\right)\left[\left(F_{1}, A\right)\right](a)(x) \oplus\left(U_{2}, A\right)\left[\left(F_{2}, A\right)\right](a)(x) \\
& =\left(\bigvee_{y \in X}\left(\left(F_{1}, A\right)(a)(y) \odot\left(U_{1}, A\right)(a)(y, x)\right)\right) \\
& \oplus\left(\bigvee_{z \in X}\left(\left(F_{2}, A\right)(a)(z) \odot\left(U_{2}, A\right)(a)(z, x)\right)\right) \\
& \geq \bigvee_{y \in X}\left\{\left(\left(F_{1}, A\right)(a)(y) \odot\left(U_{1}, A\right)(a)(y, x)\right)\right. \\
& \left.\oplus\left(\left(F_{2}, A\right)(a)(y) \odot\left(U_{2}, A\right)(a)(y, x)\right)\right\} \\
& (\text { by Lemma } 2(16)) \\
& \geq \bigvee_{y \in X}\left(\left(\left(F_{1}, A\right)(a) \oplus\left(F_{2}, A\right)\right)(a)(y)\right. \\
& \left.\odot\left(\left(U_{1}, A\right)(a) \odot\left(U_{2}, A\right)\right)(a)(y, x)\right) \\
& =\left(\left(U_{1}, A\right) \odot\left(U_{2}, A\right)\right)\left[\left(F_{1}, A\right) \oplus\left(F_{2}, A\right)\right](a)(x) .
\end{aligned}
$$

(6) and (8) are similarly proved as (5) and (7), respectively.

Theorem 14. Let $(X, A, \mathcal{U})$ be a soft $L$-fuzzy pre-uniform space. Define mappings $\xi_{\mathcal{U}}^{r}, \xi_{\mathcal{U}}^{l}: S(X, A) \times S(X, A) \rightarrow L$ by

$$
\begin{aligned}
& \xi_{\mathcal{U}}^{r}((F, A),(G, A))=\bigvee\{\mathcal{U}((U, A)) \mid(U, A)[(F, A)] \leq(G, A)\} \\
& \xi_{\mathcal{U}}^{l}((F, A),(G, A))=\bigvee\{\mathcal{U}((U, A)) \mid(U, A)[[(F, A)]] \leq(G, A)\}
\end{aligned}
$$

Then $\xi_{\mathcal{U}}^{r}$ and $\xi_{\mathcal{U}}^{l}$ are soft $L$-fuzzy bitopogenous orders. If $(X, A, \mathcal{U})$ be a soft $L$-fuzzy quasi-uniform space, then $\xi_{\mathcal{U}}^{r}$ and $\xi_{\mathcal{U}}^{l}$ are soft $L$-fuzzy bitopogenous stuctures.

Proof. $\quad(\mathrm{ST} 1)$ Since $(U, A)\left[\left(0_{X}, A\right)\right]=\left(0_{X}, A\right)$ and $(U, A)\left[\left(1_{X}, A\right)\right]=$ $\left(1_{X}, A\right)$, for $\mathcal{U}((U, A))=1$, we have

$$
\xi_{\mathcal{U}}^{r}\left(\left(0_{X}, A\right),\left(0_{X}, A\right)\right)=\xi_{\mathcal{U}}^{r}\left(\left(1_{X}, A\right),\left(1_{X}, A\right)\right)=1
$$

(ST2) If $\xi_{\mathcal{U}}^{r}((F, A),(G, A)) \neq 0$, there exists $(U, A) \in S(X \times X, A)$ with $\mathcal{U}((U, A))>0$. By Lemma $13(1),(F, A) \leq(U, A)[(F, A)] \leq(G, A)$. Hence $(F, A) \leq(G, A)$.

(ST3) If $\left(F_{1}, A\right) \leq(F, A),(G, A) \leq\left(G_{1}, A\right)$, then

$$
\begin{aligned}
\xi_{\mathcal{U}}^{r}((F, A),(G, A)) & =\bigvee\{\mathcal{U}((U, A)) \mid(U, A)[(F, A)] \leq(G, A)\} \\
& \leq \bigvee\left\{\mathcal{U}((U, A)) \mid(U, A)[(F, A)] \leq\left(G_{1}, A\right)\right\} \\
& \leq \bigvee\left\{\mathcal{U}((U, A)) \mid(U, A)\left[\left(F_{1}, A\right)\right] \leq\left(G_{1}, A\right)\right\} \\
& =\xi_{\mathcal{U}}^{r}\left(\left(F_{1}, A\right),\left(G_{1}, A\right)\right)
\end{aligned}
$$


(T) By Lemma 13(5),

$$
\begin{aligned}
& \xi_{\mathcal{U}}^{r}\left(\left(F_{1}, A\right),\left(G_{1}, A\right)\right) \odot \xi_{\mathcal{U}}^{r}\left(\left(F_{2}, A\right),\left(G_{2}, A\right)\right) \\
& =\bigvee\left\{\mathcal{U}((U, A)) \mid(U, A)\left[\left(F_{1}, A\right)\right] \leq\left(G_{1}, A\right)\right\} \\
& \odot \bigvee\left\{\mathcal{U}((V, A)) \mid(V, A)\left[\left(F_{2}, A\right)\right] \leq\left(G_{2}, A\right)\right\} \\
& \leq \bigvee\left\{\mathcal{U}((U, A)) \odot \mathcal{U}((V, A)) \mid(U, A)\left[\left(F_{1}, A\right)\right]\right. \\
& \left.\odot(V, A)\left[\left(F_{2}, A\right)\right] \leq\left(G_{1}, A\right) \odot\left(G_{2}, A\right)\right\} \\
& \leq \bigvee\{\mathcal{U}((U, A) \odot(V, A)) \mid((U, A) \odot(V, A)) \\
& \left.\left[\left(F_{1}, A\right) \odot\left(F_{2}, A\right)\right] \leq\left(G_{1}, A\right) \odot\left(G_{2}, A\right)\right\} \\
& \leq \bigvee\left\{\mathcal{U}((W, A)) \mid(W, A)\left[\left(F_{1}, A\right) \odot\left(F_{2}, A\right)\right] \leq\left(G_{1}, A\right) \odot\left(G_{2}, A\right)\right\} \\
& =\xi_{\mathcal{U}}\left(\left(F_{1}, A\right) \odot\left(F_{2}, A\right),\left(G_{1}, A\right) \odot\left(G_{2}, A\right)\right) .
\end{aligned}
$$

(CT) By Lemma 13(7),

$$
\begin{aligned}
& \xi_{\mathcal{U}}^{r}\left(\left(F_{1}, A\right),\left(G_{1}, A\right)\right) \odot \xi_{\mathcal{U}}^{r}\left(\left(F_{2}, A\right),\left(G_{2}, A\right)\right) \\
& =\bigvee\left\{\mathcal{U}((U, A)) \mid(U, A)\left[\left(F_{1}, A\right)\right] \leq\left(G_{1}, A\right)\right\} \\
& \odot \bigvee\left\{\mathcal{U}((V, A)) \mid(V, A)\left[\left(F_{2}, A\right)\right] \leq\left(G_{2}, A\right)\right\} \\
& \leq \bigvee\left\{\mathcal{U}((U, A)) \odot \mathcal{U}((V, A)) \mid(U, A)\left[\left(F_{1}, A\right)\right]\right. \\
& \left.\oplus(V, A)\left[\left(F_{2}, A\right)\right] \leq\left(G_{1}, A\right) \oplus\left(G_{2}, A\right)\right\} \\
& \leq \bigvee\{\mathcal{U}((U, A) \odot(V, A)) \mid((U, A) \odot(V, A)) \\
& \left.[(F, A) \oplus(F, A)] \leq\left(G_{1}, A\right) \oplus\left(G_{2}, A\right)\right\} \\
& \leq \bigvee\left\{\mathcal{U}((W, A)) \mid(W, A)[(F, A)] \leq\left(G_{1}, A\right) \odot\left(G_{2}, A\right)\right\} \\
& =\xi_{\mathcal{U}}^{r}\left(\left(F_{1}, A\right) \odot\left(F_{2}, A\right),\left(G_{1}, A\right) \odot\left(G_{2}, A\right)\right) .
\end{aligned}
$$

Hence $\xi_{\mathcal{U}}^{r}$ is a soft $L$-fuzzy bitopogenous order. Similarly, $\xi_{\mathcal{U}}^{r}$ is a soft $L$-fuzzy bitopogenous order.

Let $(X, A, \mathcal{U})$ be a soft $L$-fuzzy quasi-uniform space. Then (TS) For each $(U, A) \in S(X \times X, A)$ such that $(U, A)[(F, A)] \leq(G, A)$, by $(\mathrm{Q})$, we have

$$
\mathcal{U}((U, A)) \leq \bigvee\{\mathcal{U}((V, A)) \odot \mathcal{U}((W, A)) \mid(V, A) \circ(W, A) \leq(U, A)\}
$$

Thus,

$$
\begin{aligned}
& \bigvee\{\mathcal{U}((U, A)) \mid(U, A)[(F, A)] \leq(G, A)\} \\
& \leq \bigvee\{\mathcal{U}((V, A)) \odot \mathcal{U}((W, A)) \mid(V, A) \circ(W, A) \\
& {[(F, A)]=(V, A)[(W, A)[(F, A)] \leq(G, A)\}} \\
& \leq \bigvee(H, A) \in S(X, A)\{\bigvee\{\mathcal{U}((V, A)) \odot \mathcal{U}((W, A)) \mid \\
& (W, A)[(F, A)] \leq(H, A),(V, A)[(H, A)] \leq(G, A)\}\} \\
& \leq \bigvee(H, A) \in S(X, A)\{\bigvee\{\mathcal{U}((V, A)) \mid(V, A)[(H, A)] \leq \\
& (G, A)\} \odot \bigvee\{\mathcal{U}((W, A)) \mid(W, A)[(F, A)] \leq(H, A)\}\} \\
& =\bigvee(H, A) \in S(X, A) \xi_{\mathcal{U}}^{r}((F, A),(H, A)) \odot \xi_{\mathcal{U}}^{r}((H, A),(G, A))
\end{aligned}
$$


Hence $\xi_{\mathcal{U}}^{r}$ is a soft $L$-fuzzy bitopogenous structure. Similarly, $\xi_{\mathcal{U}}^{l}$ is a soft $L$-fuzzy bitopogenous structure.

Example 15. Let $H, E$ and $([0,1], \wedge, \rightarrow, 0,1)$ as Example 9. Let $A=$ $\{b, c\} \subset E$ and $X=\left\{h^{1}, h^{4}, h^{5}\right\} \subset H$. Put a fuzzy soft set $(U, A)$ as follow:

$$
\begin{array}{lccc}
(U,\{b\})= & & (U,\{c\})= \\
\left(\begin{array}{cccc}
b & h^{1} & h^{4} & h^{5} \\
h^{1} & 1 & 0.6 & 0.7 \\
h^{4} & 0.1 & 1 & 0.5 \\
h^{5} & 0.4 & 0.6 & 1
\end{array}\right) \quad\left(\begin{array}{cccc}
c & h^{1} & h^{4} & h^{5} \\
h^{1} & 1 & 0.3 & 0.6 \\
h^{4} & 0.1 & 1 & 0.6 \\
h^{5} & 0.7 & 0.5 & 1
\end{array}\right)
\end{array}
$$

Then we obtain $(U \odot U, A)$ as

$$
\begin{array}{ll}
(U \odot U,\{b\})= & (U \odot U,\{c\})= \\
\left(\begin{array}{cccc}
b & h^{1} & h^{4} & h^{5} \\
h^{1} & 1 & 0.2 & 0.4 \\
h^{4} & 0 & 1 & 0 \\
h^{5} & 0 & 0.2 & 1
\end{array}\right) \quad\left(\begin{array}{cccc}
c & h^{1} & h^{4} & h^{5} \\
h^{1} & 1 & 0 & 0.2 \\
h^{4} & 0 & 1 & 0.2 \\
h^{5} & 0.4 & 0 & 1
\end{array}\right)
\end{array}
$$

Define $\mathcal{U}: S(X \times X, A) \rightarrow L$ as follows

$$
\mathcal{U}((V, A))= \begin{cases}1, & \text { if }(V, A)=\left(\top_{X \times X}, A\right), \\ 0.6, & \text { if }(U, A) \leq(V, A) \neq\left(\top_{X \times X}, A\right), \\ 0.3, & \text { if }(U, A) \odot(U, A) \leq(V, A) \geq(U, A), \\ 0, & \text { otherwise. }\end{cases}
$$

Since $0.3=\mathcal{U}(((W \odot W), A) \geq \mathcal{U}((W, A)) \odot \mathcal{U}((W, A))=0.2, \mathcal{U}$ is a soft $L$ fuzzy pre-uniformity on $X$. But $\mathcal{U}$ is not a soft $L$-fuzzy quasi-uniformity on $X$ because

$$
\begin{aligned}
& 0.3=\mathcal{U}((U, A) \odot(U, A)) \\
& \not \leq \bigvee\{\mathcal{U}((V, A)) \odot \mathcal{U}((W, A)) \mid(V, A) \circ(W, A) \leq(U, A) \odot(U, A)\} \\
& =0
\end{aligned}
$$

By Theorem 14, we obtain soft $L$-fuzzy bitopogenous orders $\xi_{\mathcal{U}}^{r}, \xi_{\mathcal{U}}^{l}: S(X, A) \times$ $S(X, A) \rightarrow L$ as follows

$$
\xi_{\mathcal{U}}^{r}((F, A),(H, A))= \begin{cases}1, & \text { if }\left(\top_{X \times X}, A\right)[F, A] \leq(H, A) \\ 0.6, & \text { if }(W, A)[(F, A)] \leq(H, A), \\ & \left(\top_{X \times X}, A\right)[F, A] \not \subset(H, A) \\ 0.3, & \text { if }(W \odot W, A)[(F, A)] \leq(H, A), \\ & (W, A)[(F, A)] \not \leq(H, A), \\ 0, & \text { otherwise },\end{cases}
$$




$$
\xi_{\mathcal{U}}^{l}((F, A),(H, A))= \begin{cases}1, & \text { if }\left(\top_{X \times X}, A\right)[[F, A]] \leq(H, A) \\ 0.6, & \text { if }(W, A)[[(F, A)] \leq(H, A), \\ & \left(\top_{X \times X}, A\right)[[F, A]] \not \leq(H, A) \\ 0.3, & \text { if }(W \odot W, A)[[(F, A)] \leq(H, A), \\ & (W, A)[[(F, A)]] \not \leq(H, A), \\ 0, & \text { otherwise. }\end{cases}
$$

But $\xi_{\mathcal{U}}^{r}$ is not a soft $L$-fuzzy bitopogenous structure because

$$
\begin{aligned}
& 0=\left(\xi_{\mathcal{U}}^{r} \circ \xi_{\mathcal{U}}^{r}\right)((W \odot W, A)[(F, A)],(W \odot W, A)[(F, A)]) \\
& \geq \xi_{\mathcal{U}}^{r}((W \odot W, A)[(F, A)],(W \odot W, A)[(F, A)])=0.3 .
\end{aligned}
$$

\section{References}

[1] K.V. Babitha, J.J. Sunil, Soft set relations and functions, Compu. Math. Appl., 60(2010), 1840-1849, doi: 10.1016/j.camwa.2010.07.014.

[2] N. Cag̈man, S. Karatas and S. Enginoglu, Soft topology, Comput. Math. Appl., 62(1) (2011), 351-358. doi: 10.1016/j.camwa.2011.05.016.

[3] F. Feng, X. Liu, V.L. Fotea, Y.B. Jun, Soft sets and soft rough sets, Information Sciences, 181 (2011), 1125-1137, doi: 10.1016/j.ins.2010.11.004.

[4] P. Hájek, Metamathematices of Fuzzy Logic, Kluwer Academic Publishers, Dordrecht (1998), doi: 10.1007/978-94-011-5300-3.

[5] U. Höhle, S.E. Rodabaugh, Mathematics of Fuzzy Sets: Logic, Topology, and Measure Theory, The Handbooks of Fuzzy Sets Series 3, Kluwer Academic Publishers, Boston, 1999, doi: 10.1007/978-1-4615-5079-2.

[6] A.K. Katsaras, On fuzzy uniform spaces, J. Math. Anal. Appl., 101, 1984, 97-113. doi: 10.1016/0022-247x(84)90060-x.

[7] A. Kharal, B. Ahmad, Mappings on soft classes, New Math. Nat. Comput., 7(3) (2011), 471-481.

[8] R. Lowen, Fuzzy uniform spaces, J. Math. Anal. Appl., 82 (1981), 370-385, doi: 10.1016/0022-247x(81)90202-x.

[9] D. Molodtsov, Soft set theory, Comput. Math. Appl., 37(1999), 19-31.

[10] Z. Pawlak, Rough sets, Int. J. Comput. Inf. Sci., 11 (1982), 341-356.

[11] Z. Pawlak, Rough probability, Bull. Pol. Acad. Sci. Math., 32(1984), 607-615.

[12] M. Shabir and M. Naz, On soft topological spaces, Comput. Math. Appl.,61 (2011), 1786-1799, doi: 10.1016/j.camwa.2011.02.006.

[13] B. Tanay and M. B. Kandemir, Topological structure of fuzzy soft sets, Comput. Math. Appl., 61(10) (2011), 2952-2957, doi: 10.1016/j.camwa.2011.03.056.

[14] Hu Zhao and Sheng-Gang Li, L-fuzzifying soft topological spaces and L-fuzzifying soft interior operators, Ann. Fuzzy Math. Inform., 5(3) (2013), 493-503.

[15] Í. Zorlutuna, M. Akdag, W. K. Min and S. Atmaca, Remarks on soft topological spaces, Ann. Fuzzy Math. Inform., 3(2) (2012), 171-185. 